\title{
Solvent-free Glycidyl Carbamates oligomerization and solvent affinity of oligomers
}

Guillaume Taing, ${ }^{1,2}$ Vanessa Legros, ${ }^{1}$ Françoise Ta, ${ }^{1}$ David Da Silva, ${ }^{1}$ Cyril Colas, ${ }^{1,3}$

Marylène Vayer, ${ }^{2}$ Marie Schuler, ${ }^{1}$ Kamel Chougrani, ${ }^{4}$ Christophe Sinturel, ${ }^{2}$ Arnaud

Tatibouet* 1

1 - Université d'Orléans, CNRS, ICOA UMR7311, Rue de Chartres, F-45067, Orléans Cedex

2, France

2 - Université d'Orléans, CNRS, ICMN UMR7374, 1 b Rue de la Férollerie, F-45071, Orléans Cedex 2, France

3 - CNRS, CBM, UPR4301, Université d'Orléans, Rue Charles Sadron, F-45071, Orléans Cedex 2, France

4 - Parfums et cosmétiques Christian DIOR, LVMH Recherche, 185 Avenue de Verdun, F45800, Saint-Jean-de-Braye, France

Corresponding author: arnaud.tatibouet@ univ-orleans.fr 


\section{CONTENTS}

MALDI-TOF analysis.

Oligoglycidylcarbamates analysis overview S4

$\operatorname{Poly}(N, N$-dimethylcarbamate) initiated with DMAP

Poly $(N, N$-dimethylcarbamate) initiated with PPY S10-S14

$\operatorname{Poly}(N, N$-dimethylcarbamate) initiated with AJ S $15-S 17$

Poly( $N$-piperidinylcarbamate) initiated with DMAP $\mathrm{S} 18-\mathrm{S} 23$

Poly $(N$-morpholinylcarbamate) initiated with DMAP. S24-S27

SEC Calibration S28

Poly(glycidyl carbamate) affinity with solvents S29-S30 
MALDI-TOF analysis of poly(glycidyl $\mathrm{N}$-dimethylcarbamate) obtained by using $\mathrm{CsCl}$ or $\mathrm{NaCl}$ as cationating agents display exactly similar $m / z$ (Figure S1), suggesting that no expected adducts are formed between the added cations and the polymer. Because MALDI-TOF is used in positive mode, a positive charged has to be present on the polymer chain, and thus the adduct should in fact be a proton $\mathrm{H}^{+}$. Hereby, we strongly suspect that $\mathrm{p}(\mathrm{GC})$ obtained by nucleophilic ring-opening polymerization using pyridine-based neutral reagents remained as a zwitterionic molecule.

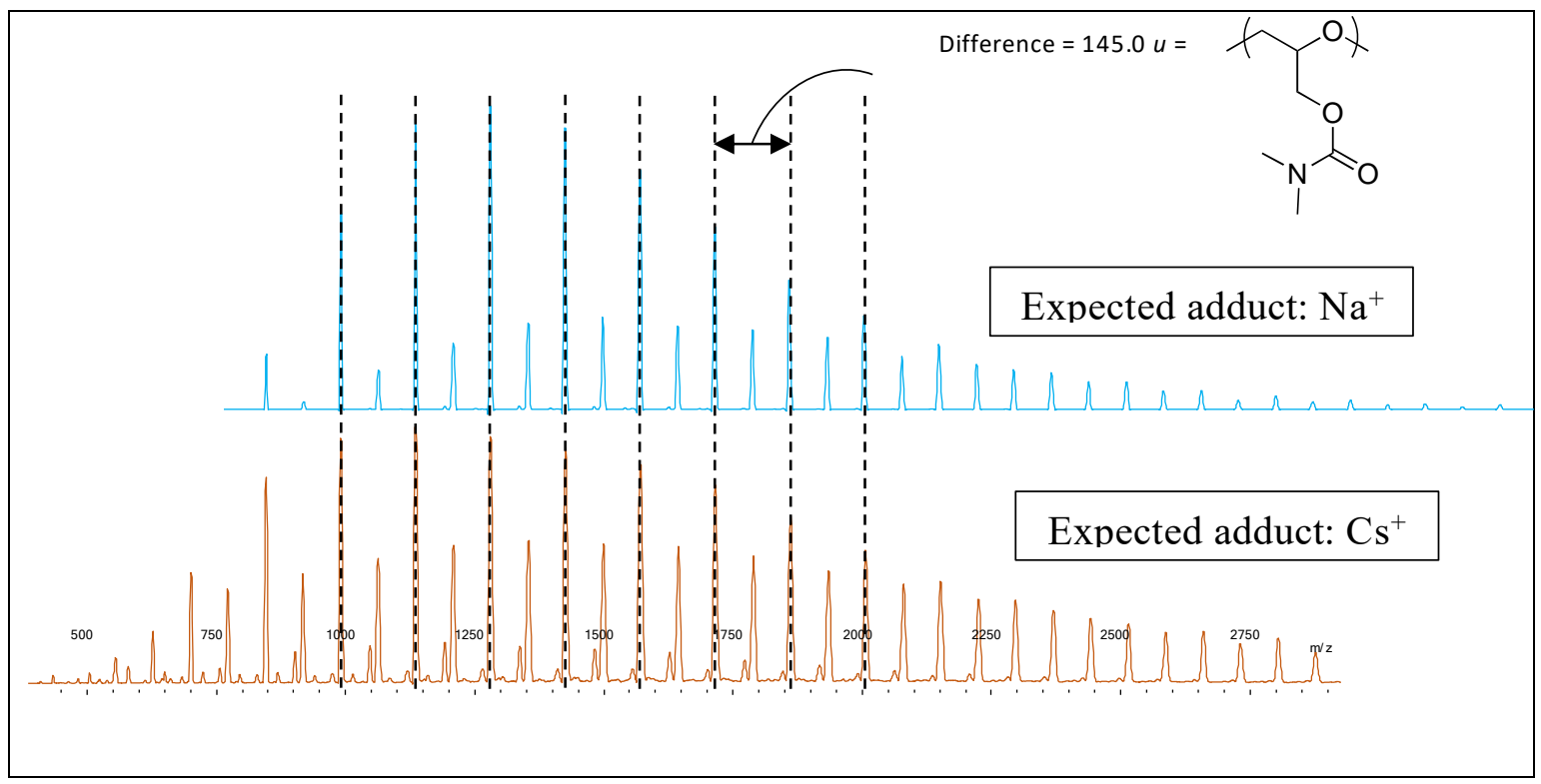

Figure S1: MALDI-TOF spectrum of poly(glycidyl N,N-dimethylcarbamate) initiated with 5 mol\% of DMAP. Cationating agent used: $\mathrm{NaCl}$ (top) or $\mathrm{CsCl}$ (bottom) 


\section{Oligoglycidylcarbamates analysis}

This section describes some chosen samples of oligoglycerol with additional elements such as

NMR spectra, SEC traces and MALDI-TOF spectra. (see Table 2, main article)

The sample are organized as follow:

- $\quad \operatorname{poly}($ glycidyl $N, N$-dimethylcarbamate) initiated with DMAP, PPY and AJ

- $\operatorname{poly(glycidyl~} N$-piperidinylcarbamate) initiated with DMAP

- $\quad \operatorname{poly}($ glycidyl $N$-morpholinylcarbamate) initiated with DMAP 
$\operatorname{Poly}(N, N$-dimethylcarbamate) initiated with DMAP (5 mol\%) (Table 2, entry 1)

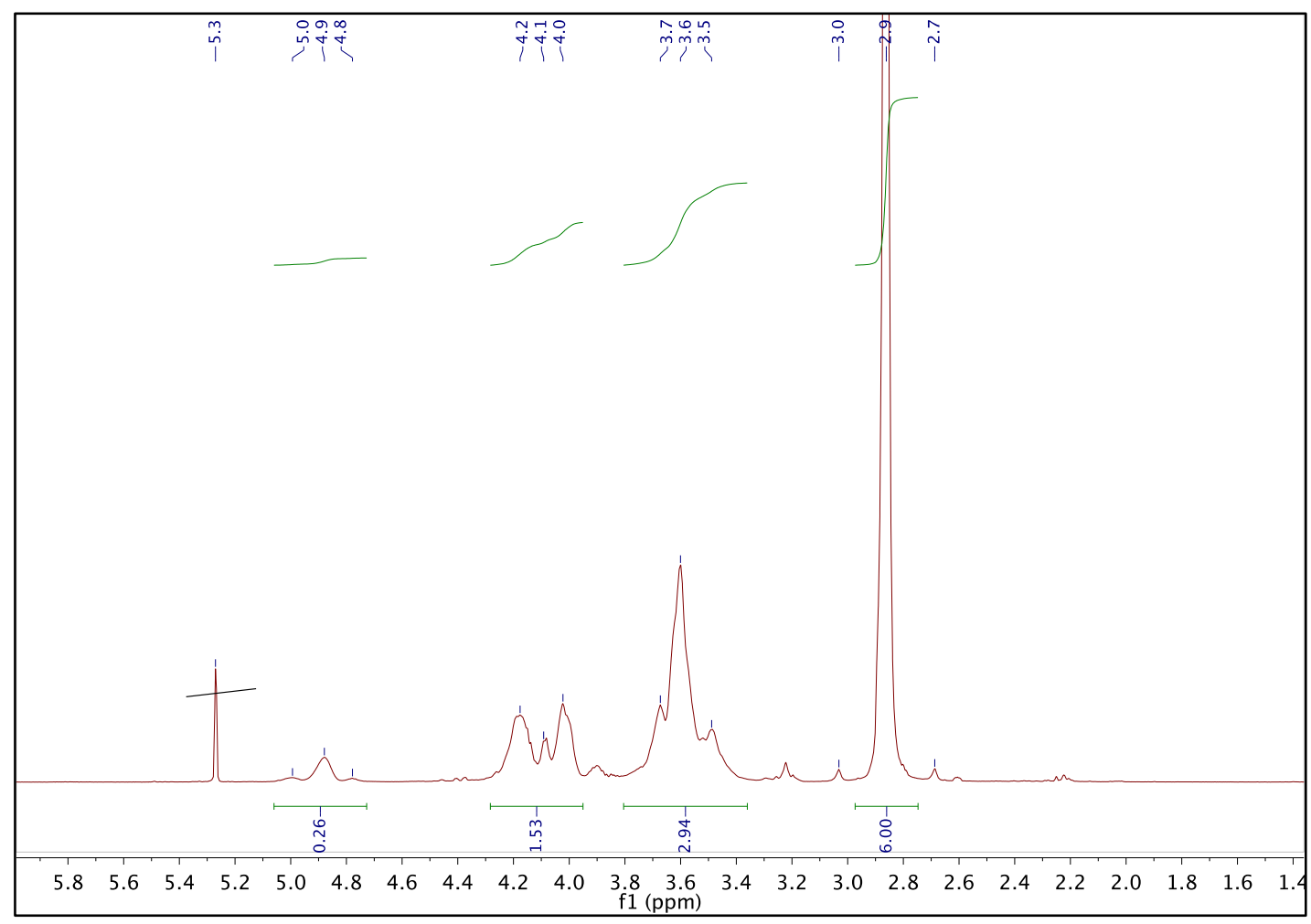

Figure S2: ${ }^{1} \mathrm{H}-\mathrm{NMR}\left(\mathrm{CDCl}_{3}\right)$ spectrum of poly(glycidyl $N, N$-dimethylcarbamate) initiated with DMAP (5 mol\%) (Table 2, entry 1) 


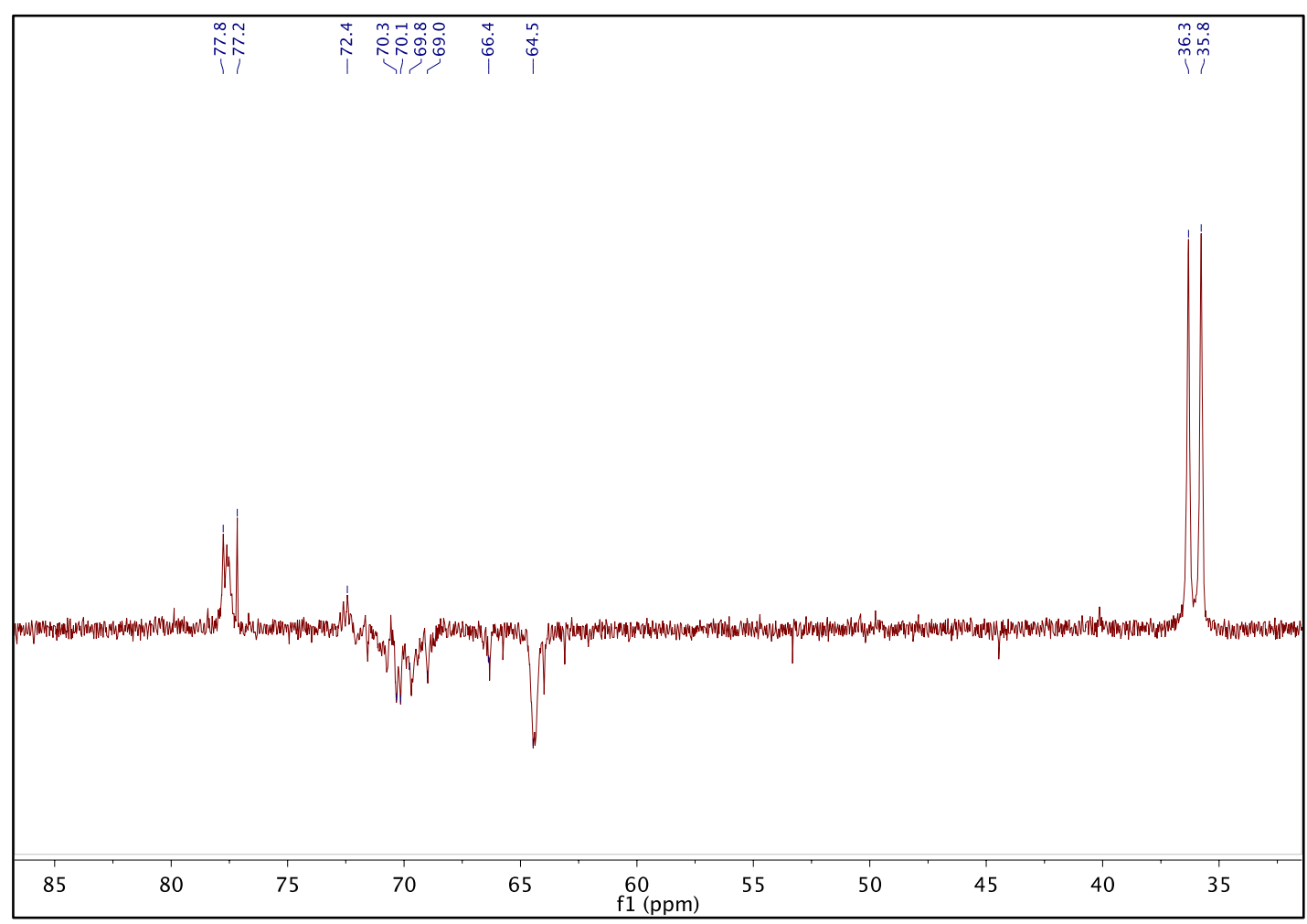

Figure S3: DEPT-NMR $\left(\mathrm{CDCl}_{3}\right)$ spectrum of poly(glycidyl $N, N$-dimethylcarbamate) initiated with DMAP (2.5 mol\%) (Table 2, entry 1) 


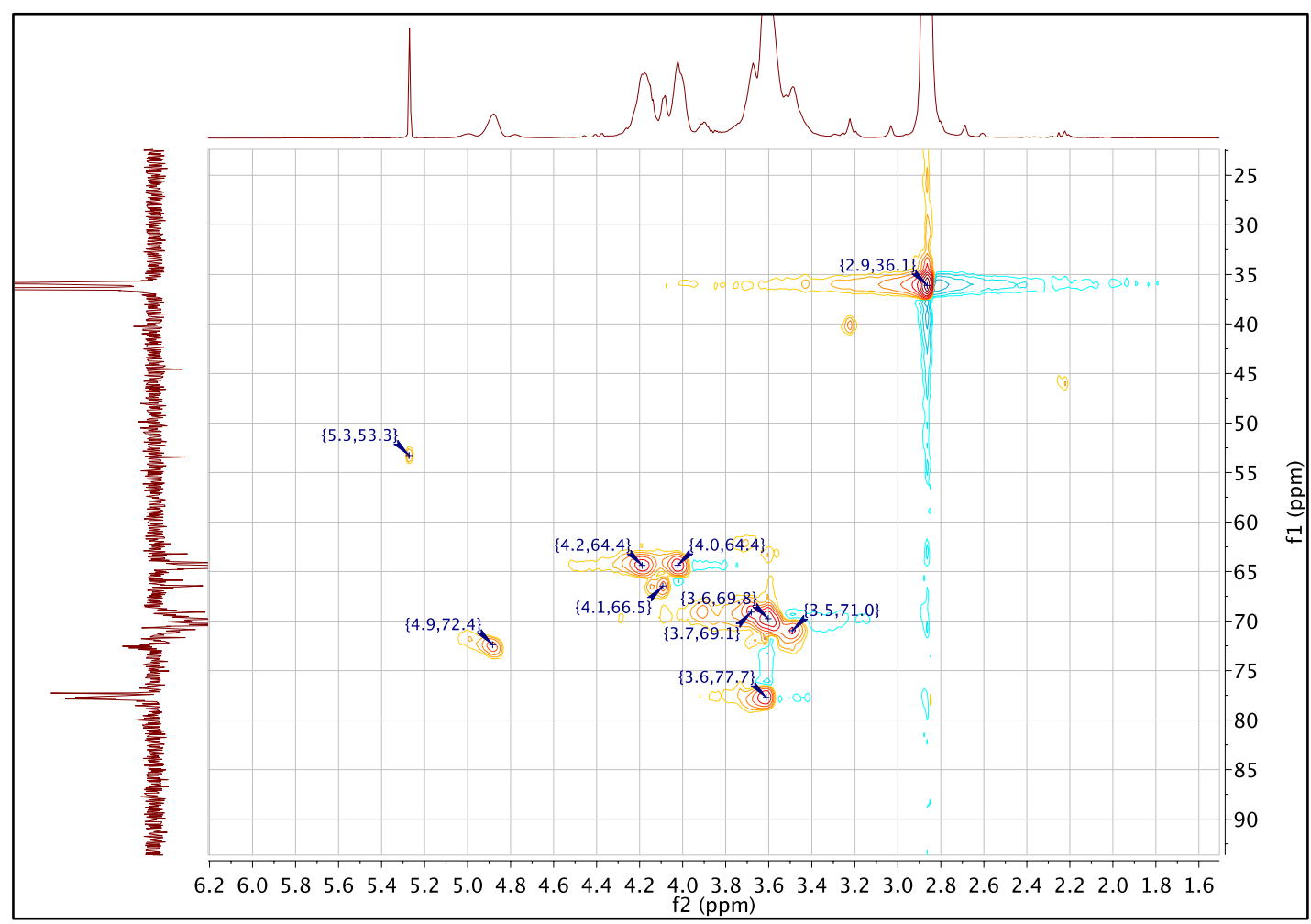

Figure S4: HSQC NMR spectrum of poly(glycidyl $N, N$-dimethylcarbamate) initiated by DMAP (5 mol\%) (Table 2, entry 1) in $\mathrm{CDCl}_{3}$, and proton assignment to the different structures determined. 


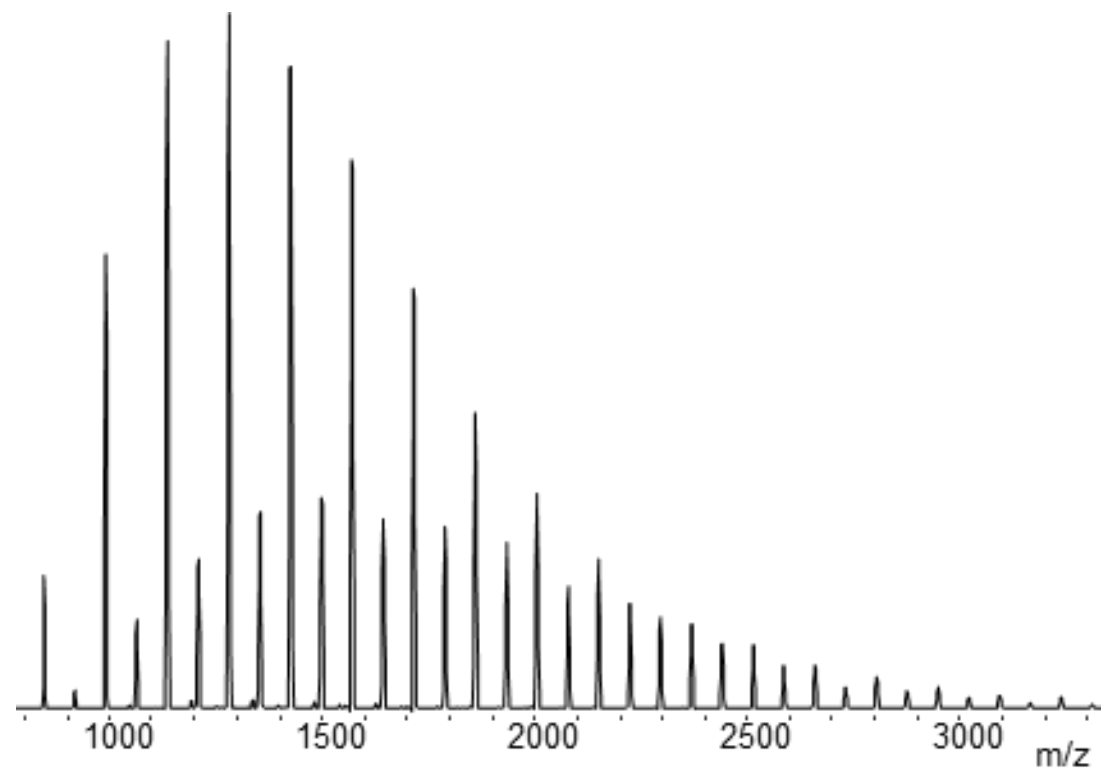

Figure S5: MALDI-TOF spectrum of poly(glycidyl $N, N$-dimethylcarbamate) initiated with DMAP (5 mol\%) (Table 2, entry 1)

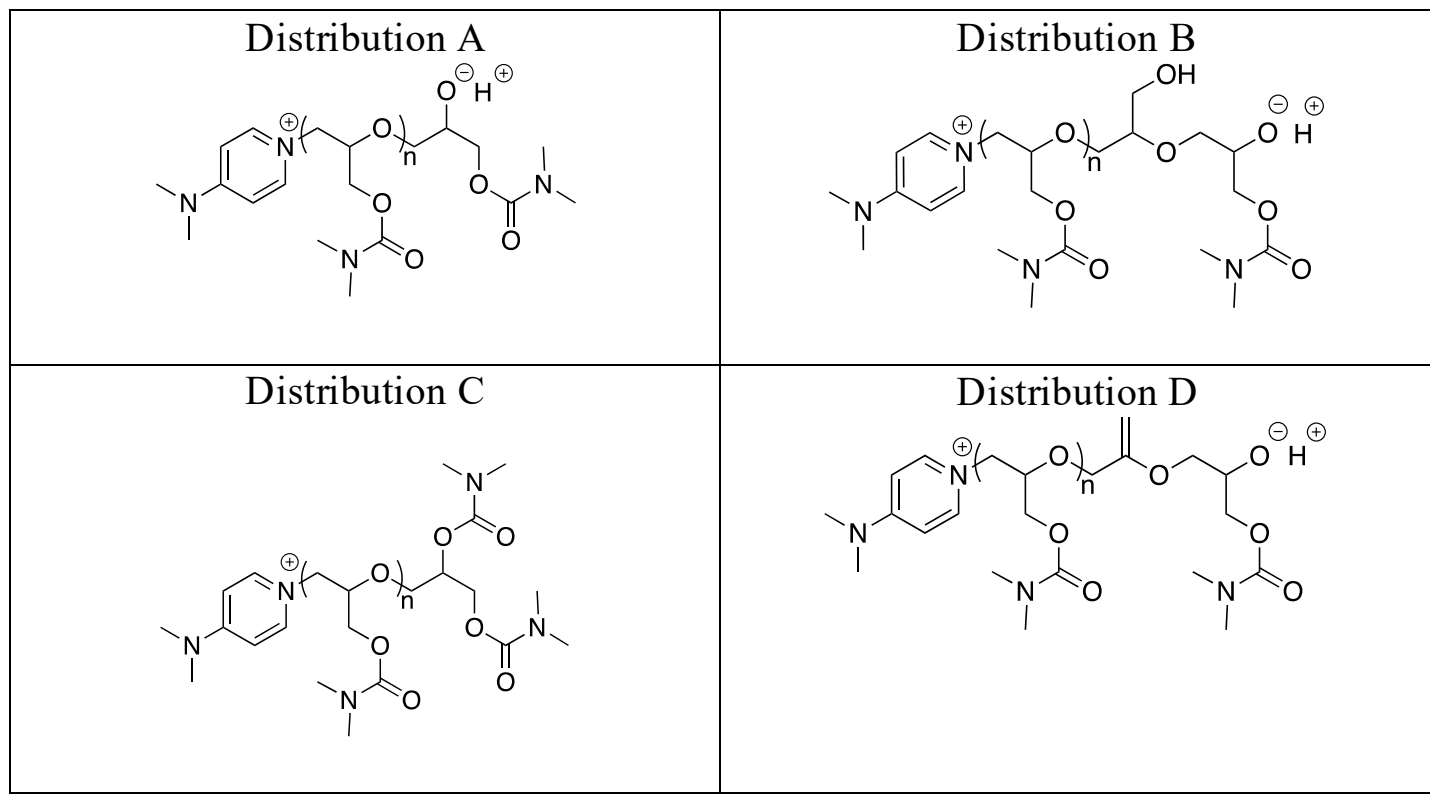

\begin{tabular}{|c|c|c|c|c|c|c|c|c|}
\hline Run & $\begin{array}{l}\left.\overline{M_{n}} \text { (theo.) }\right) \\
\left(\text { g.mol }^{-1}\right)\end{array}$ & $\overline{D P}_{\text {(theo.) }}$ & Distribution & $\begin{array}{c}\overline{\bar{M}_{n}} \\
\text { MaLDI-ToF } \\
\left(\mathbf{g} \cdot \mathbf{m o l} \mathbf{l}^{-1}\right)\end{array}$ & $\begin{array}{c}\overline{\boldsymbol{D P}} \\
\text { MALDI-ToF } \\
\end{array}$ & $\begin{array}{c}\overline{M_{n}} \\
\operatorname{sEc}^{\mathrm{e}} \\
\left(\mathrm{gemol}^{1}\right)\end{array}$ & 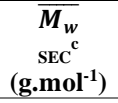 & $\mathbf{D}_{\mathrm{SEC}}$ \\
\hline \multirow{3}{*}{1} & \multirow{3}{*}{2900} & \multirow{3}{*}{20} & A & 1670 & 11 & \multirow{3}{*}{1460} & \multirow{3}{*}{2070} & \multirow{3}{*}{1.4} \\
\hline & & & $\mathrm{B} / \mathrm{C}$ & 1870 & 13 & & & \\
\hline & & & D & 1520 & 10 & & & \\
\hline
\end{tabular}

Figure S6: MALDI-TOF and SEC results of poly(glycidyl $N, N$-dimethylcarbamate) initiated with DMAP (5 mol\%) (Table 2, entry 1) 


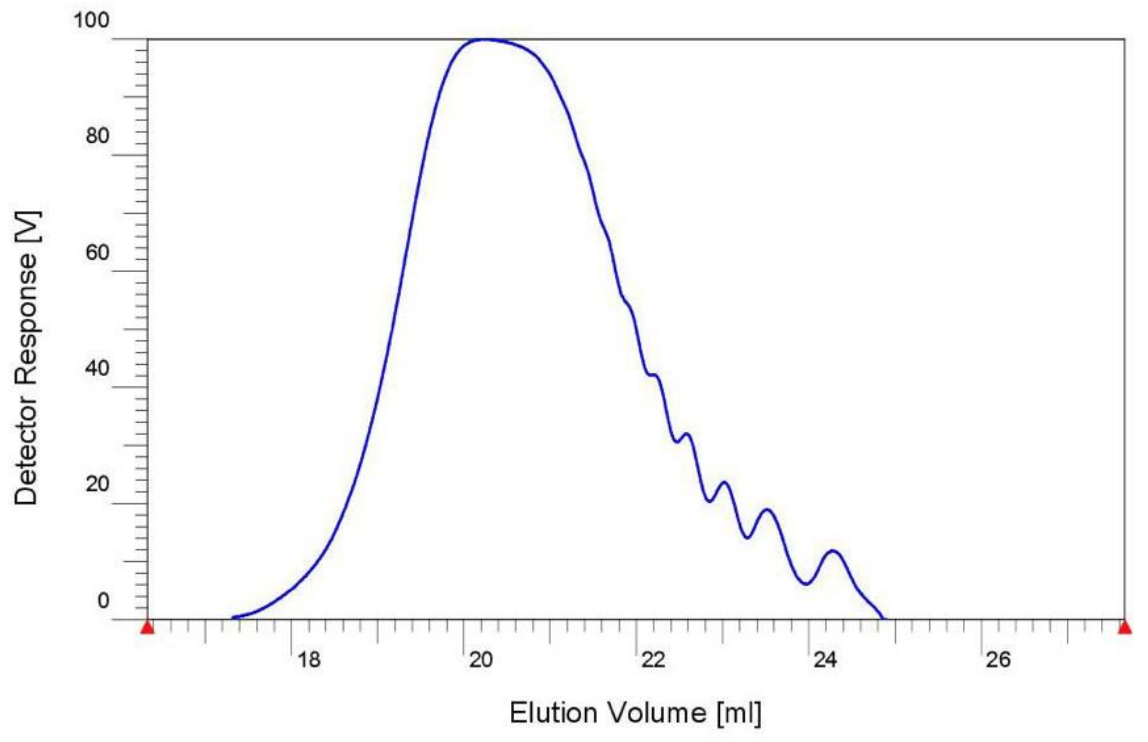

Figure S7: SEC trace of poly(glycidyl $N, N$-dimethylcarbamate) initiated with DMAP (5 mol\%) (Table 2, entry 1) 
$\operatorname{Poly}(N, N$-dimethylcarbamate) initiated with PPY (5 mol\%) (Table 2, entry 3)

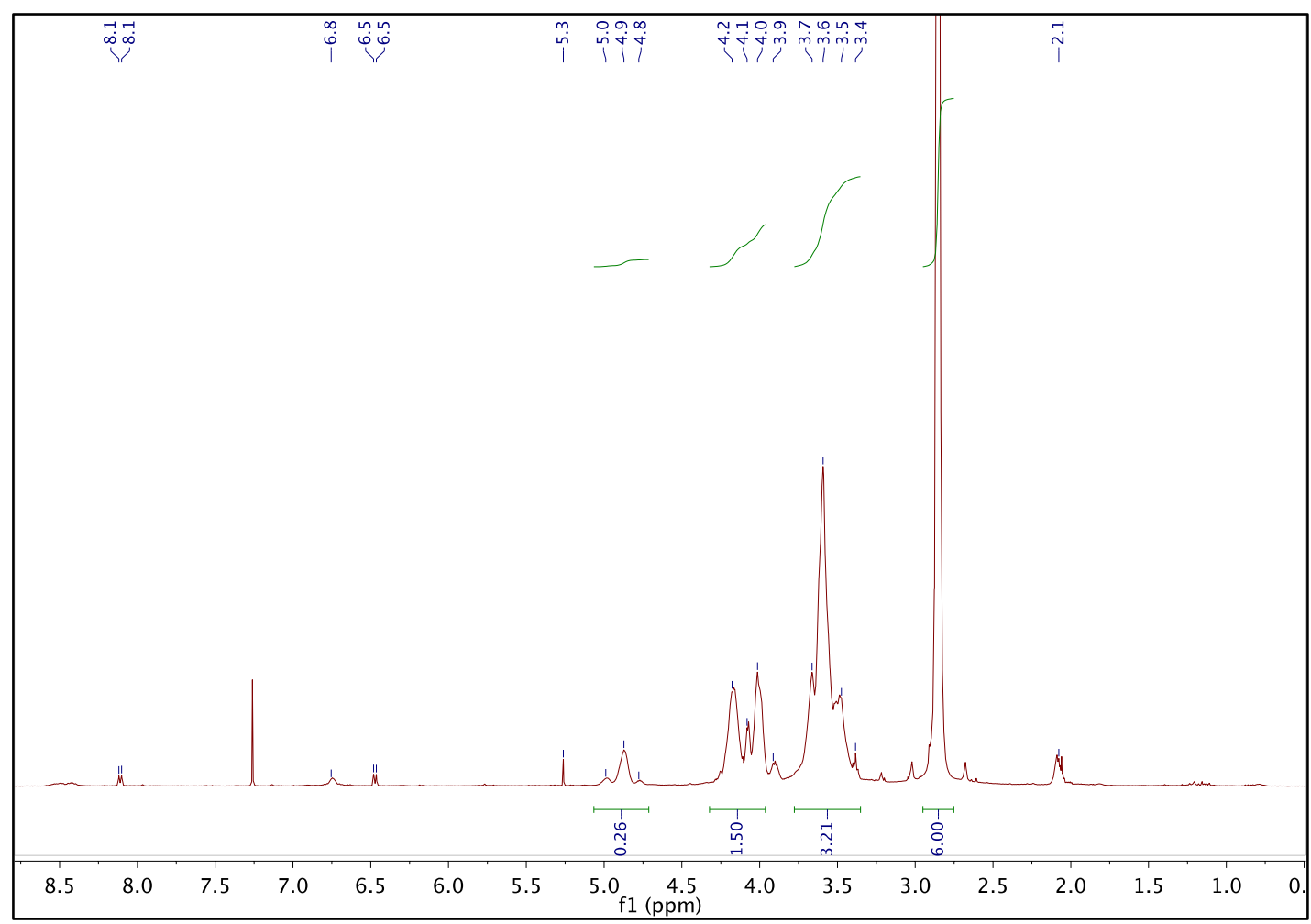

Figure S8: ${ }^{1} \mathrm{H}-\mathrm{NMR}\left(\mathrm{CDCl}_{3}\right)$ spectrum of poly(glycidyl $N, N$-dimethylcarbamate) initiated with PPY (5 mol\%) (Table 2, entry 3) 


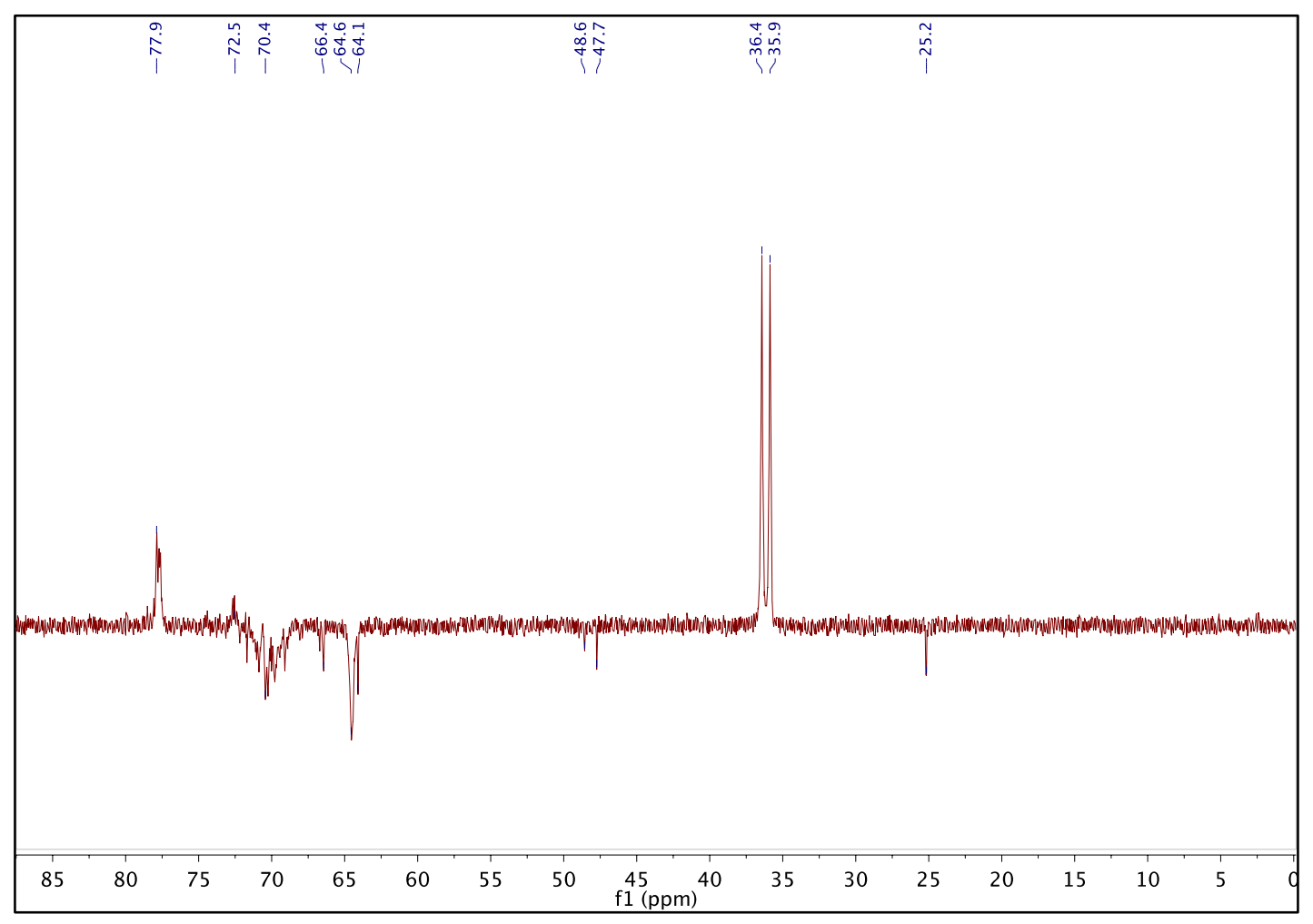

Figure S9: DEPT-NMR $\left(\mathrm{CDCl}_{3}\right)$ spectrum of poly(glycidyl $N, N$-dimethylcarbamate) initiated with PPY (5 mol\%) (Table 2, entry 3) 


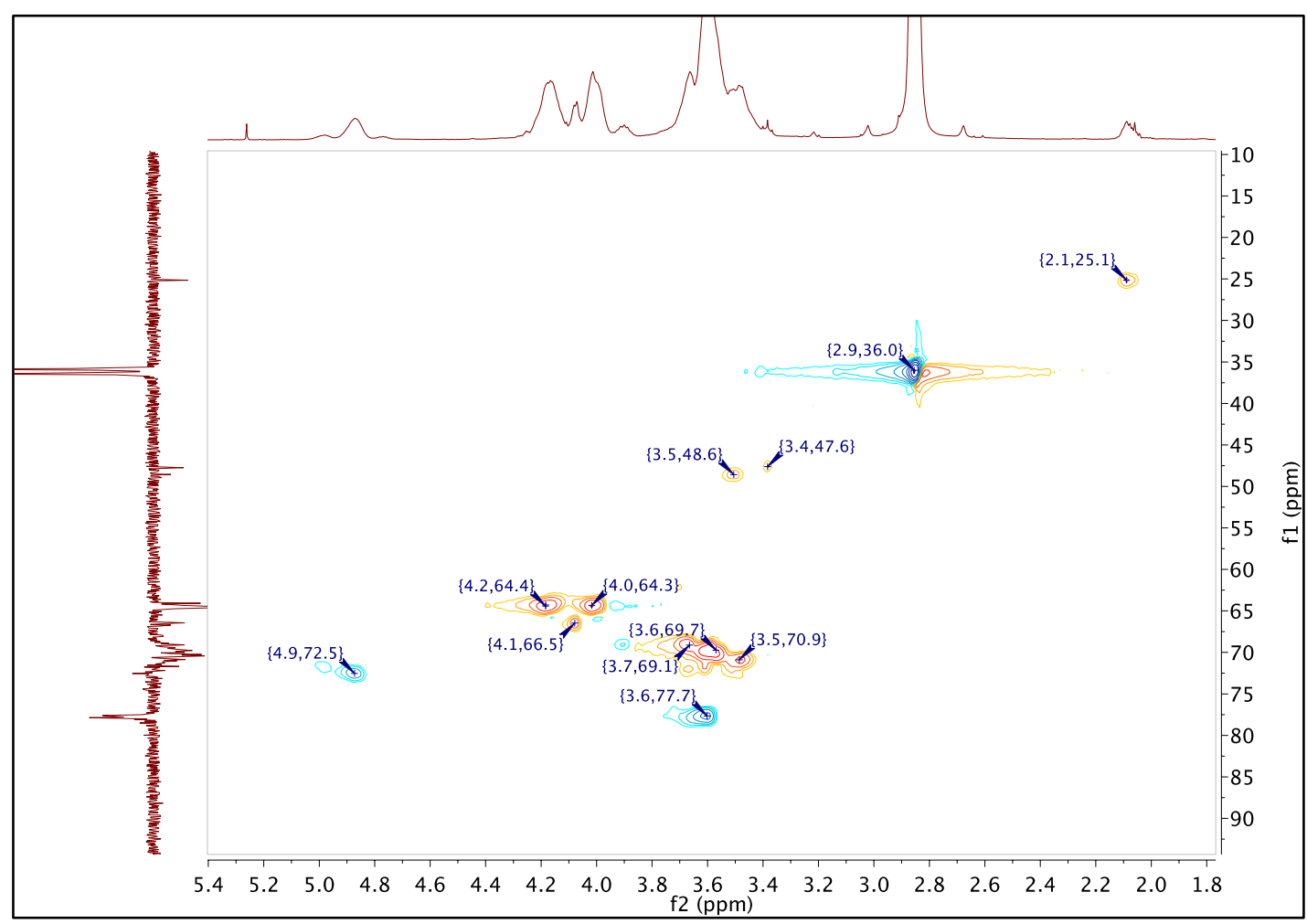

Figure S10: HSQC-NMR $\left(\mathrm{CDCl}_{3}\right)$ spectrum of poly(glycidyl $N, N$-dimethylcarbamate) initiated with PPY (5 mol\%) (Table 2, entry 3) 


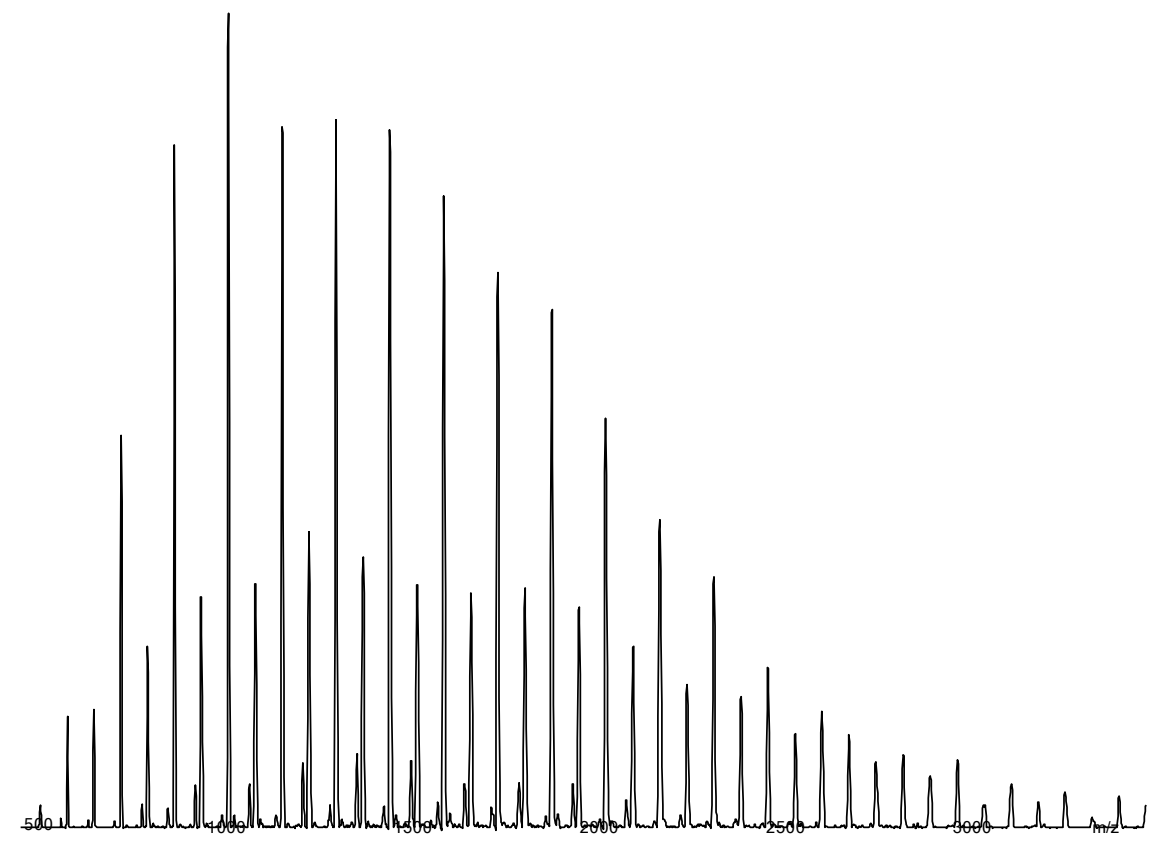

Figure S11: MALDI-TOF spectrum of poly(glydicyl $N, N$-dimethylcarbamate) initiated with PPY (5 mol\%) (Table 2, entry 3)

(n)




\begin{tabular}{|c|c|c|c|c|c|c|c|c|}
\hline Run & $\begin{array}{l}\overline{M_{n}} \text { (theo.) } \\
\left(\mathrm{g} \cdot \mathrm{mol}^{-1}\right)\end{array}$ & $\overline{D P}_{\text {(theo.) }}$ & Distribution & $\begin{array}{c}\overline{M_{n}} \text { MALDI- } \\
\text { ToF }^{\text {b }} \\
(\text { g.mol } \\
\text { (-1) }\end{array}$ & 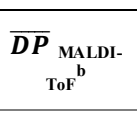 & $\begin{array}{l}\overline{M_{n}} \mathrm{SEC}^{\mathrm{c}} \\
\left(\mathrm{g} \cdot \mathrm{mol}^{-1}\right)\end{array}$ & $\begin{array}{l}\overline{M_{w}} \mathrm{SEC}^{\mathrm{c}} \\
\left(\mathrm{g} \cdot \mathrm{mol}^{-1}\right)\end{array}$ & $\boldsymbol{\boxplus}_{\mathrm{SEC}}$ \\
\hline \multirow{3}{*}{3} & \multirow{3}{*}{3050} & \multirow{3}{*}{20} & A & 1630 & 11 & \multirow{3}{*}{1580} & \multirow{3}{*}{2240} & \multirow{3}{*}{1.4} \\
\hline & & & $\mathrm{B} / \mathrm{C}$ & 1850 & 12 & & & \\
\hline & & & $\mathrm{D}$ & 1480 & 10 & & & \\
\hline
\end{tabular}

Figure S12: MALDI-TOF and SEC results of poly(glydicyl $N, N$-dimethylcarbamate) initiated with PPY (5 mol\%) (Table 2, entry 3)

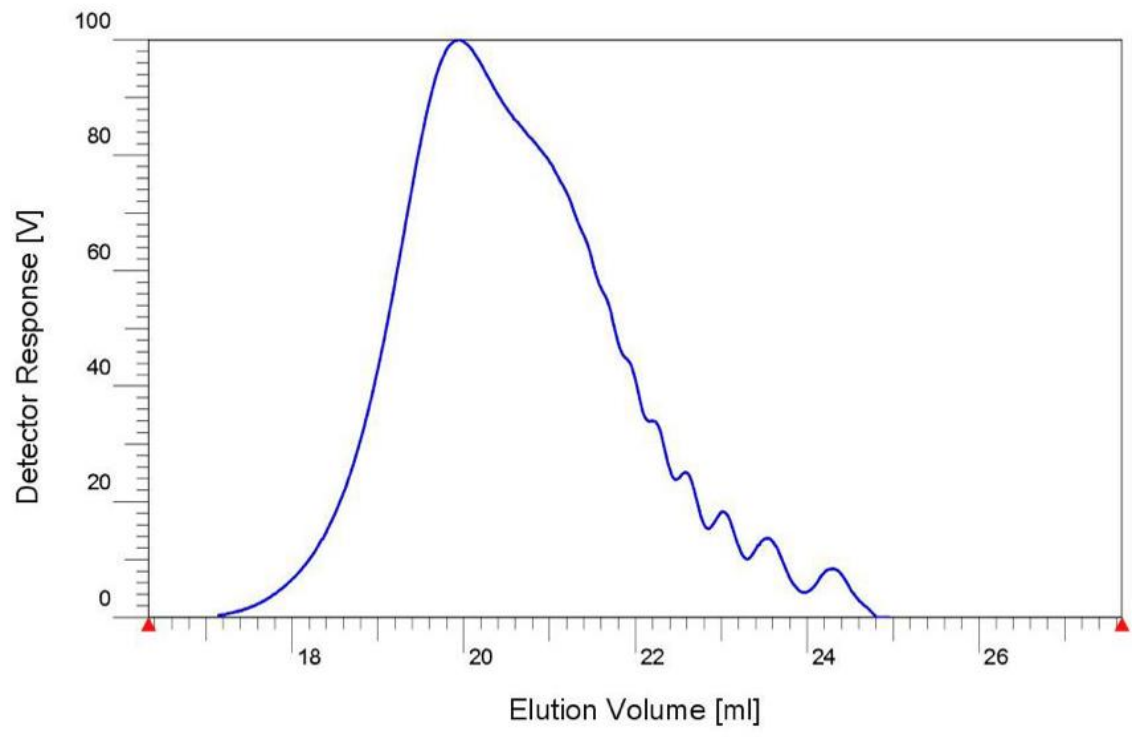

Figure S13: SEC trace of poly(glydicyl $N, N$-dimethylcarbamate) initiated with PPY (5 mol\%)

(Table 2, entry 3) 
poly(glycidyl N,N-dimethylcarbamate) initiated with AJ (5 mol\%) (Table 2, entry 5)

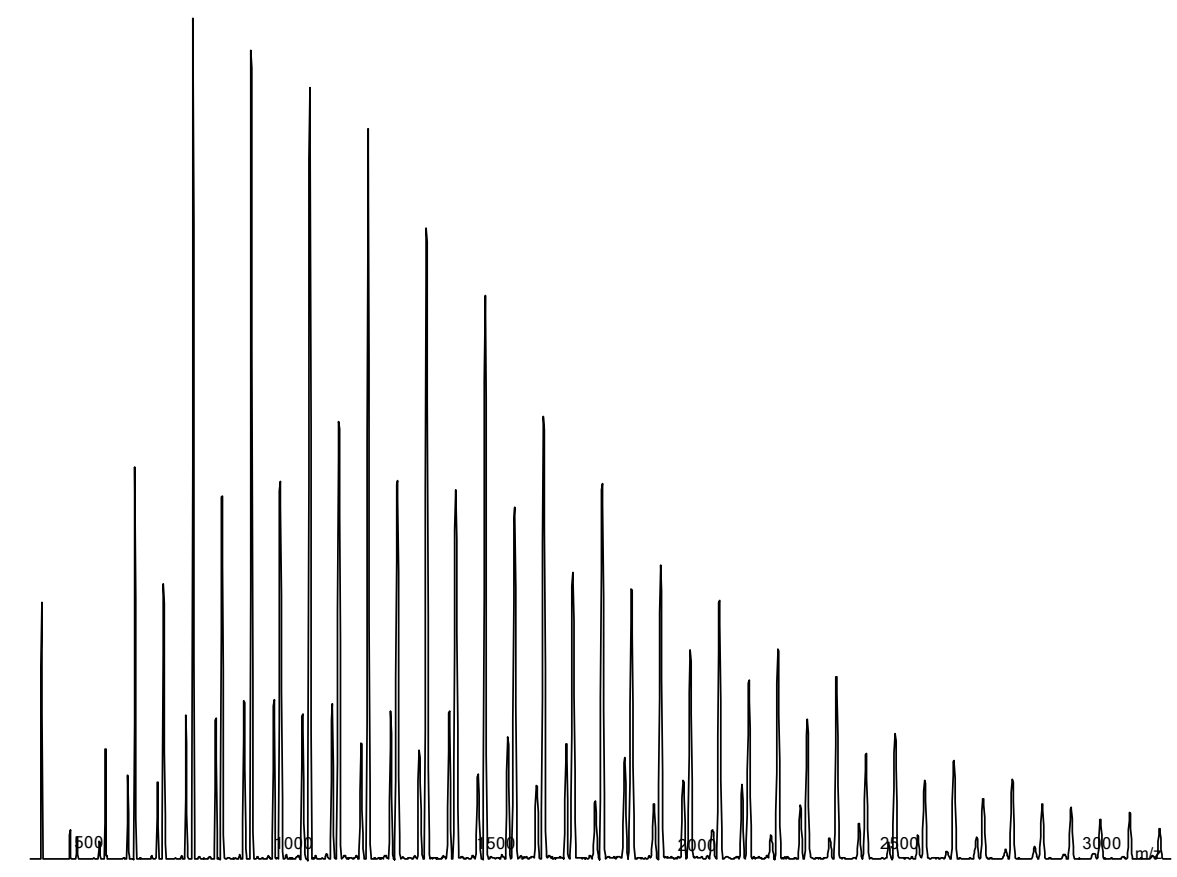

Figure S14: MALDI-TOF spectrum of poly(glycidyl $N, N$-dimethylcarbamate) initiated with AJ (5 mol\%) (Table 2, entry 5) 


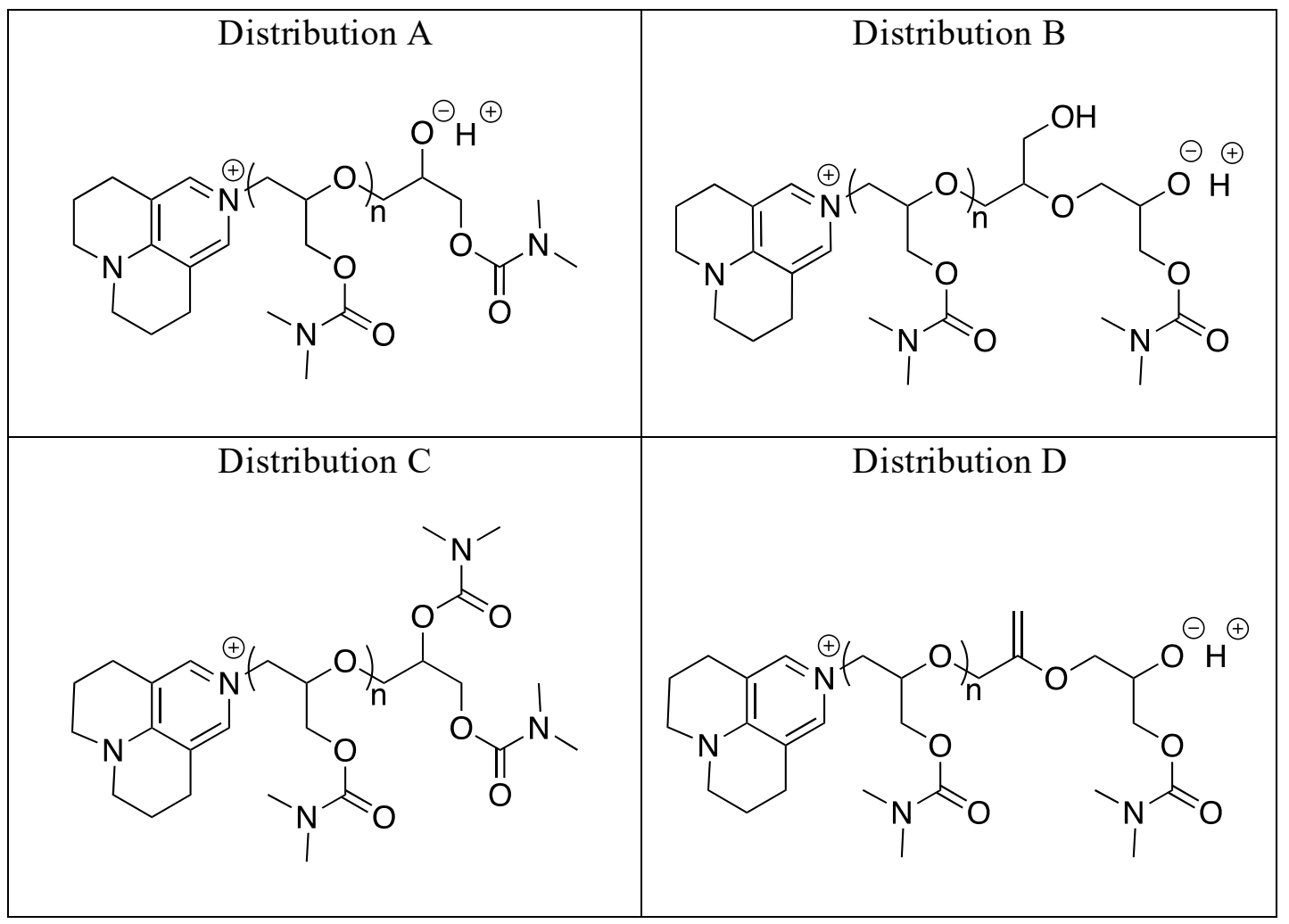

\begin{tabular}{|c|c|c|c|c|c|c|c|c|}
\hline Run & $\begin{array}{l}\overline{M_{n}} \text { (theo.) } \\
\left({\left.\mathrm{g} . \mathrm{mol}^{-1}\right)}^{-1}\right)\end{array}$ & $\overline{D P}_{\text {(theo.) }}$ & Distribution & $\begin{array}{c}\overline{M_{n}} \text { MALDI- } \\
\text { ToF } \\
\left(\mathrm{g} \cdot \mathrm{mol}^{-1}\right) \\
\end{array}$ & $\begin{array}{c}\overline{D P} \\
\mathrm{ToF}^{\text {MALDI- }} \\
\end{array}$ & $\begin{array}{l}\overline{M_{n}} \mathrm{SEC}^{\mathrm{c}} \\
\left(\mathrm{g} \cdot \mathrm{mol}^{-1}\right)\end{array}$ & $\begin{array}{l}\overline{M_{w}} \text { SEC }^{\mathrm{c}} \\
\left(\mathrm{g} \cdot \mathrm{mol}^{-1}\right)\end{array}$ & $\bigoplus_{\mathrm{SEC}}$ \\
\hline \multirow{4}{*}{5} & \multirow{4}{*}{3075} & \multirow{4}{*}{20} & A & 1540 & 10 & \multirow{4}{*}{2300} & \multirow{4}{*}{3720} & \multirow{4}{*}{1.6} \\
\hline & & & $\mathrm{B} / \mathrm{C}$ & 1620 & 11 & & & \\
\hline & & & $\mathrm{D}$ & 1480 & 10 & & & \\
\hline & & & $E^{*}$ & 1350 & 9 & & & \\
\hline
\end{tabular}

$\overline{\text { Figure S15: MALDI-TOF and SEC results of poly(glycidyl } N, N \text {-dimethylcarbamate) initiated }}$ with AJ (5 mol\%) (Table 2, entry 5) 


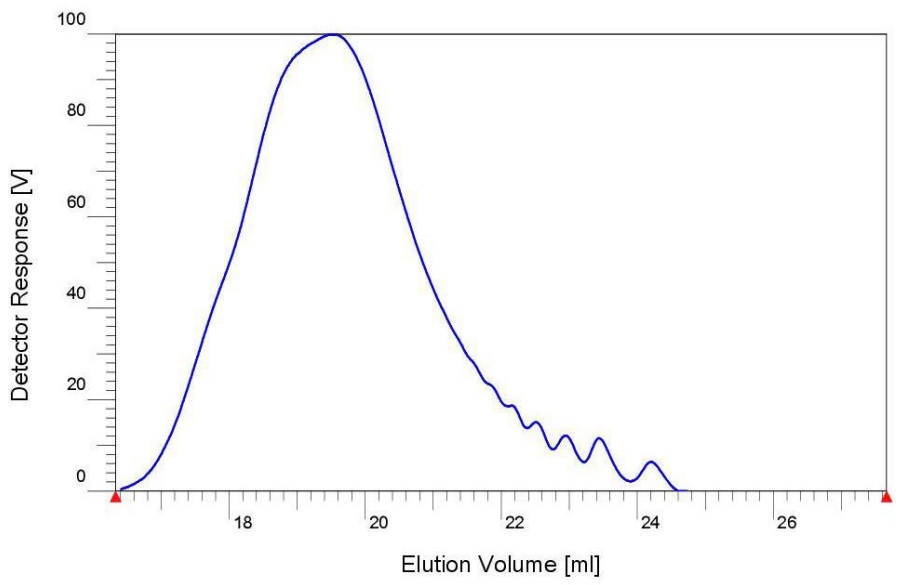

Figure S16: SEC trace of poly(glycidyl $N, N$-dimethylcarbamate) initiated with AJ (5 mol\%) (Table 2, entry 5) 
poly(glycidyl $N$-piperidinylcarbamate) initiated with DMAP (5 mol\%) (Table 2, entry 7)

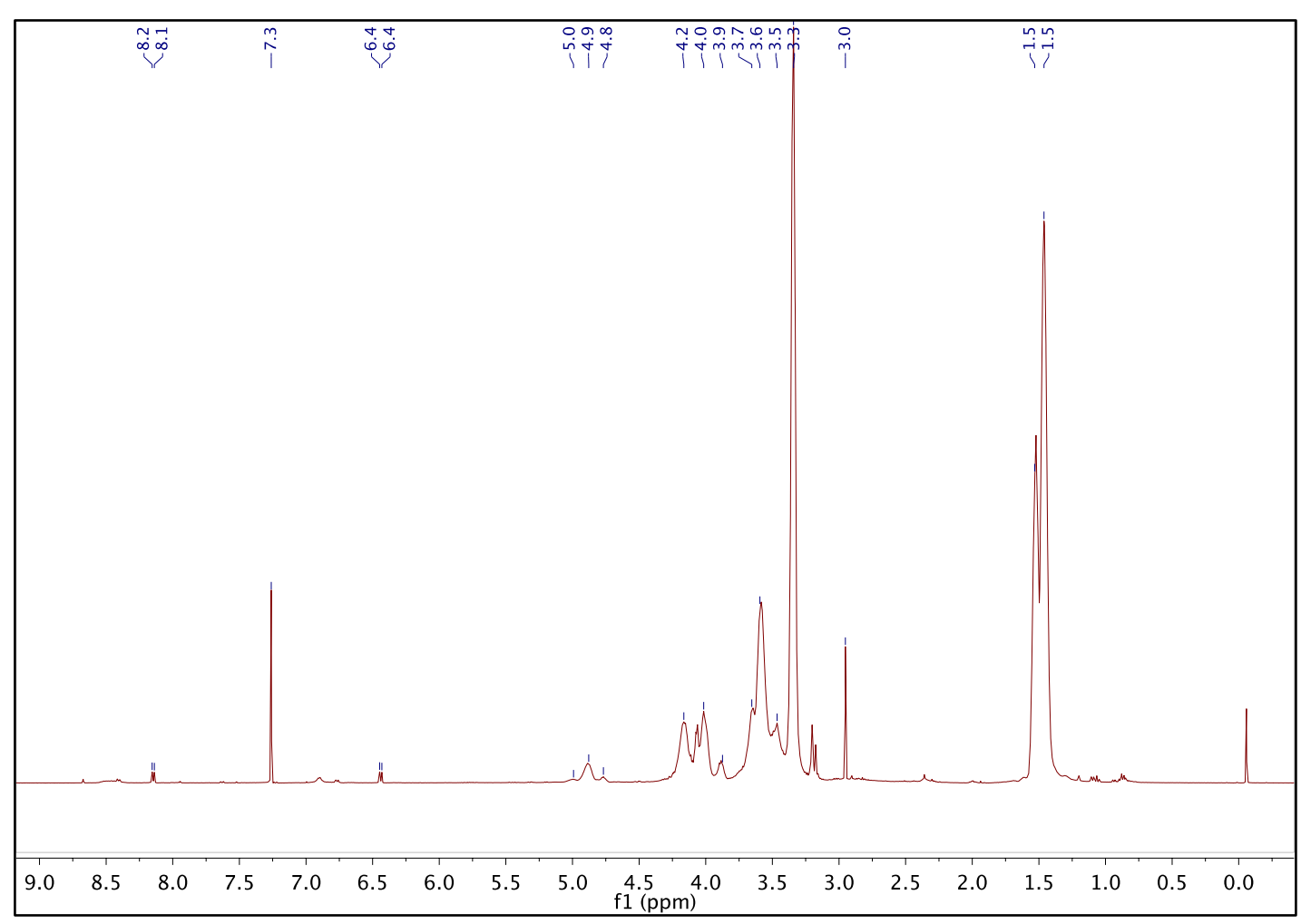

Figure S17: ${ }^{1} \mathrm{H}-\mathrm{NMR}\left(\mathrm{CDCl}_{3}\right)$ spectrum of poly(glycidyl $N$-piperidinylcarbamate) initiated with DMAP (5 mol\%) (Table 2, entry 7) 


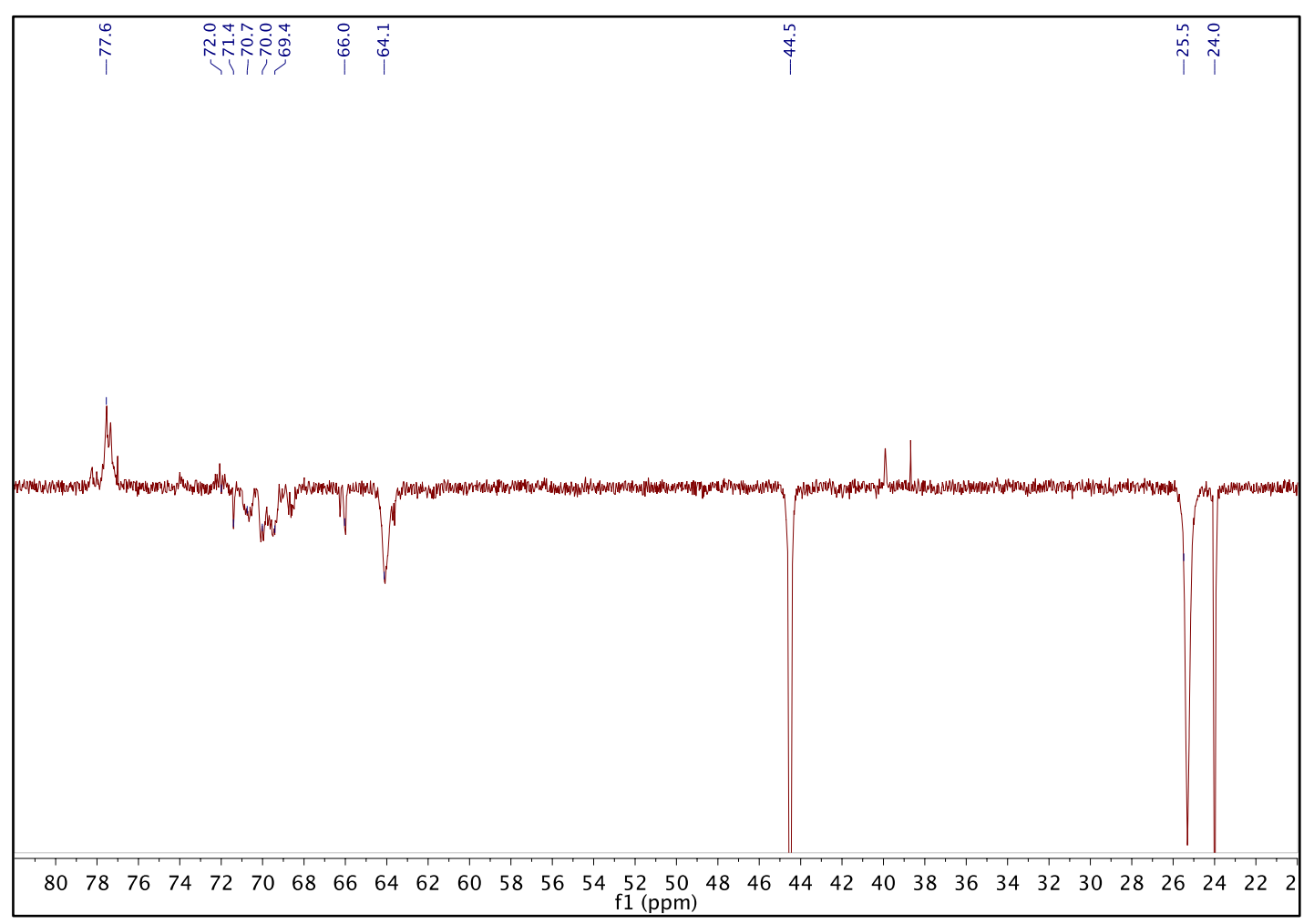

Figure S18: DEPT-NMR ( $\left.\mathrm{CDCl}_{3}\right)$ spectrum of poly(glycidyl $N$-piperidinylcarbamate) initiated with DMAP (5 mol\%) (Table 2, entry 7) 


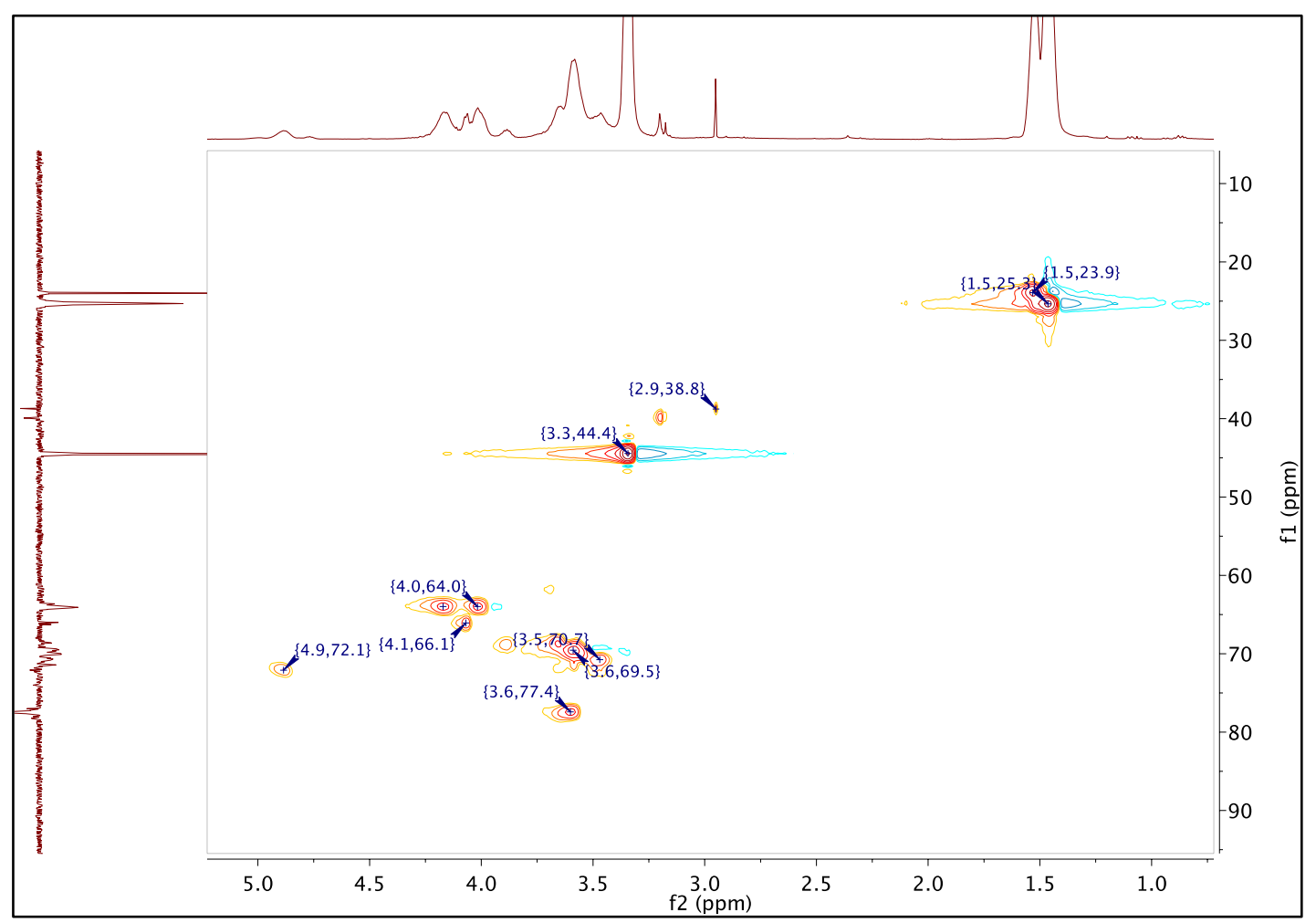

Figure S19: HSQC-NMR ( $\left.\mathrm{CDCl}_{3}\right)$ spectrum of poly(glycidyl $N$-piperidinylcarbamate) initiated with DMAP (5 mol\%) (Table 2, entry 7) 


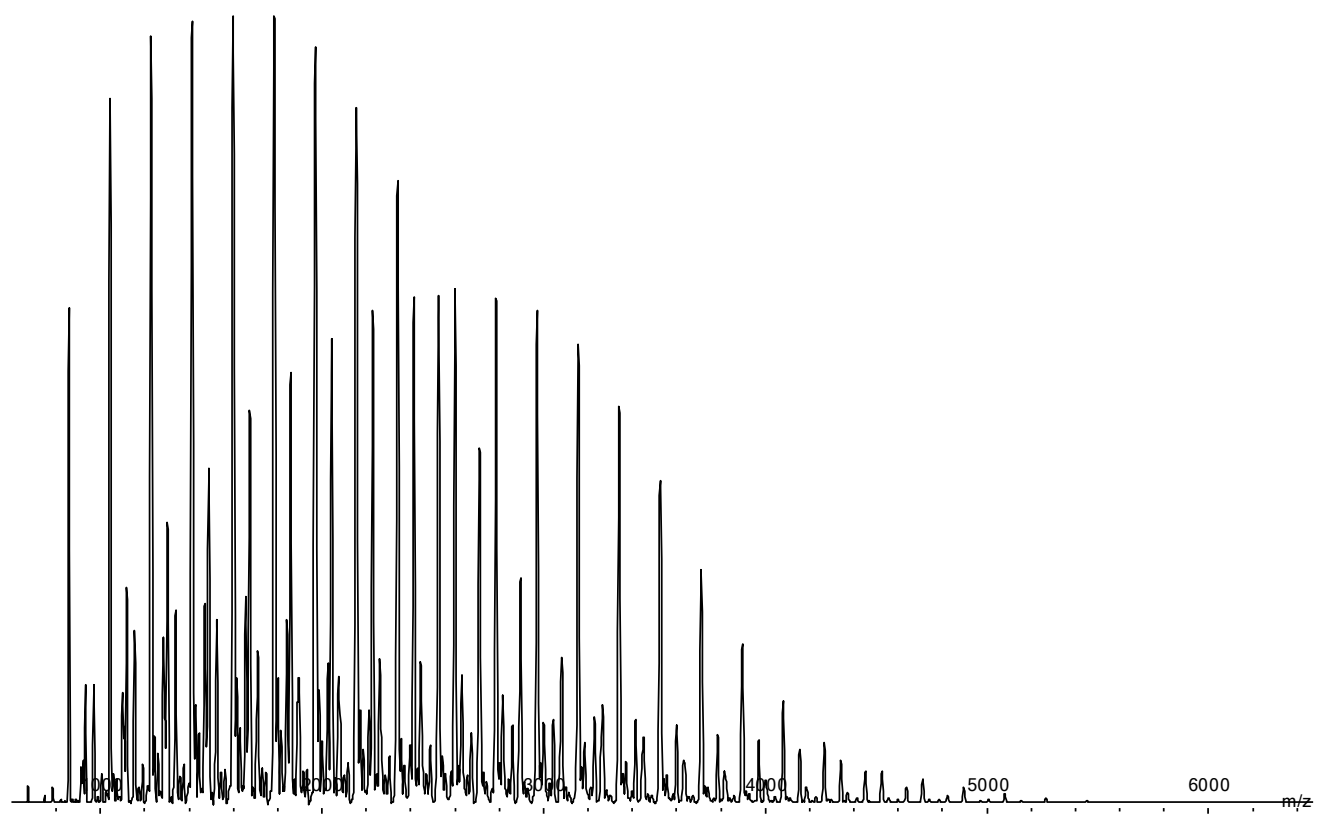

Figure S20: MALDI-TOF spectrum of poly(glycidyl $N$-piperidinylcarbamate) initiated with DMAP (5 mol\%) (Table 2, entry 7)

(n)




\begin{tabular}{|c|c|c|c|c|c|c|c|c|}
\hline entry & $\begin{array}{l}\overline{M_{n}} \text { (theo.) } \\
\left.\text { (g.mol }{ }^{-1}\right)\end{array}$ & $\overline{D P}_{\text {(theo.) }}$ & Distribution & 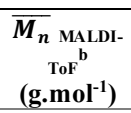 & $\begin{array}{c}\overline{D P}_{\text {MALDI- }} \\
\text { ToF }^{\text {b }} \\
\end{array}$ & $\begin{array}{c}\overline{M_{n}} \mathrm{SEC}^{\mathrm{c}} \\
\left(\mathrm{g} \cdot \mathrm{mol}^{-1}\right)\end{array}$ & $\begin{array}{c}\overline{M_{w}} \text { SEC }^{\mathrm{c}} \\
\left(\mathrm{g} \cdot \mathrm{mol}^{-1}\right)\end{array}$ & $\boldsymbol{\boxplus}_{\mathrm{SEC}}$ \\
\hline \multirow{5}{*}{7} & \multirow{5}{*}{3825} & \multirow{5}{*}{20} & A & 1920 & 10 & \multirow{5}{*}{1400} & \multirow{5}{*}{1830} & \multirow{5}{*}{1.4} \\
\hline & & & B & 2580 & 14 & & & \\
\hline & & & $\mathrm{C}$ & 1740 & 9 & & & \\
\hline & & & $\mathrm{D}$ & 1500 & 8 & & & \\
\hline & & & $\mathrm{E}$ & 3180 & 17 & & & \\
\hline
\end{tabular}

Figure S21: MALDI-TOF and SEC results of poly(glycidyl $N$-piperidinylcarbamate) initiated with DMAP (5 mol\%) (Table 2, entry 7) 


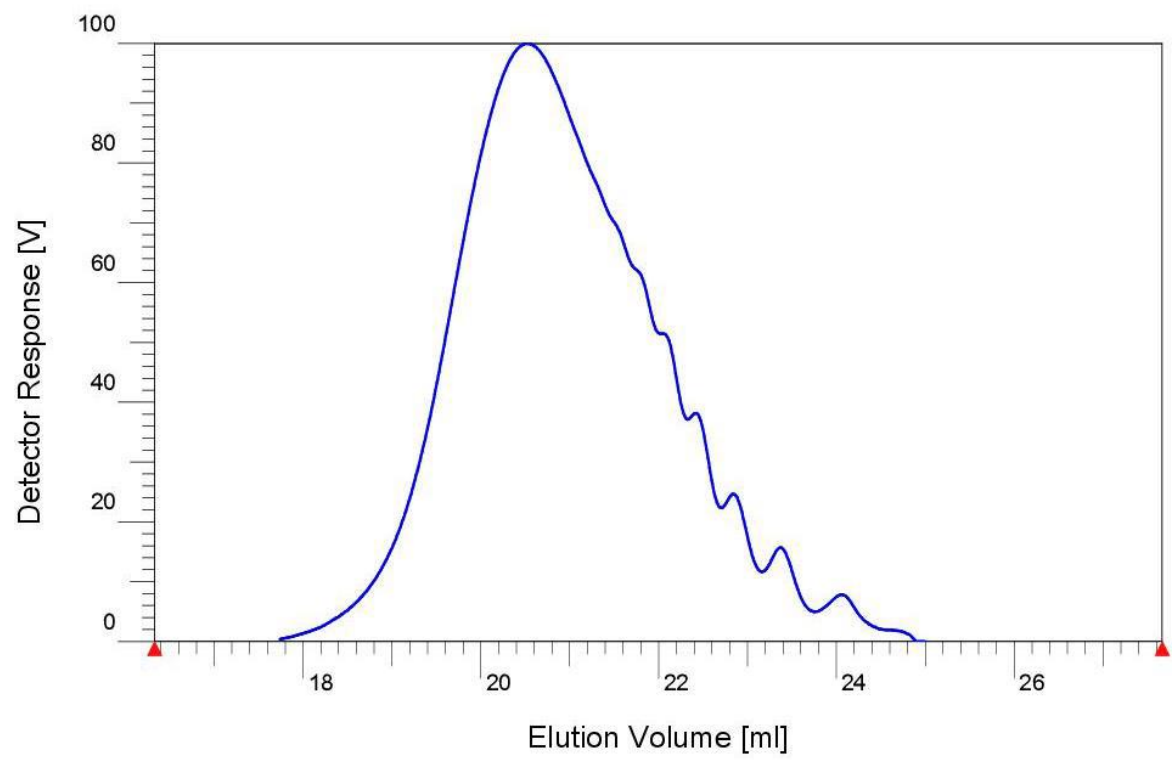

Figure S22: SEC trace of poly(glycidyl $N$-piperidinylcarbamate) initiated with DMAP (5 mol\%) (Table 2, entry 7) 
poly(glycidyl $N$-morpholinylcarbamate) initiated with DMAP (5 mol\%) (Table 2, run 13)

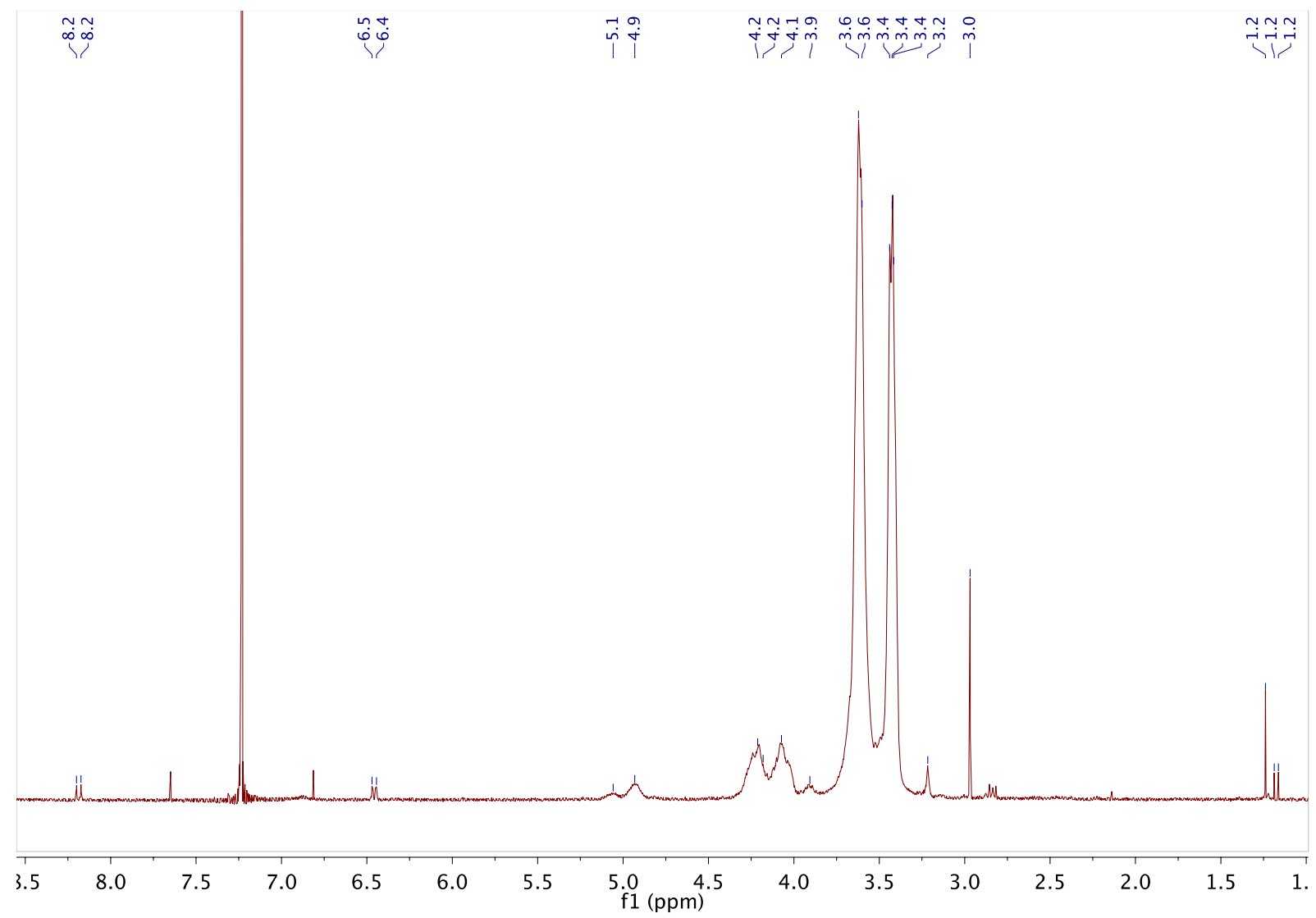

Figure S23: ${ }^{1} \mathrm{H}-\mathrm{NMR}\left(\mathrm{CDCl}_{3}\right)$ spectrum of poly(glycidyl $N$-dimethylcarbamate) initiated with DMAP (5 mol\%) (Table 2, entry 13) 

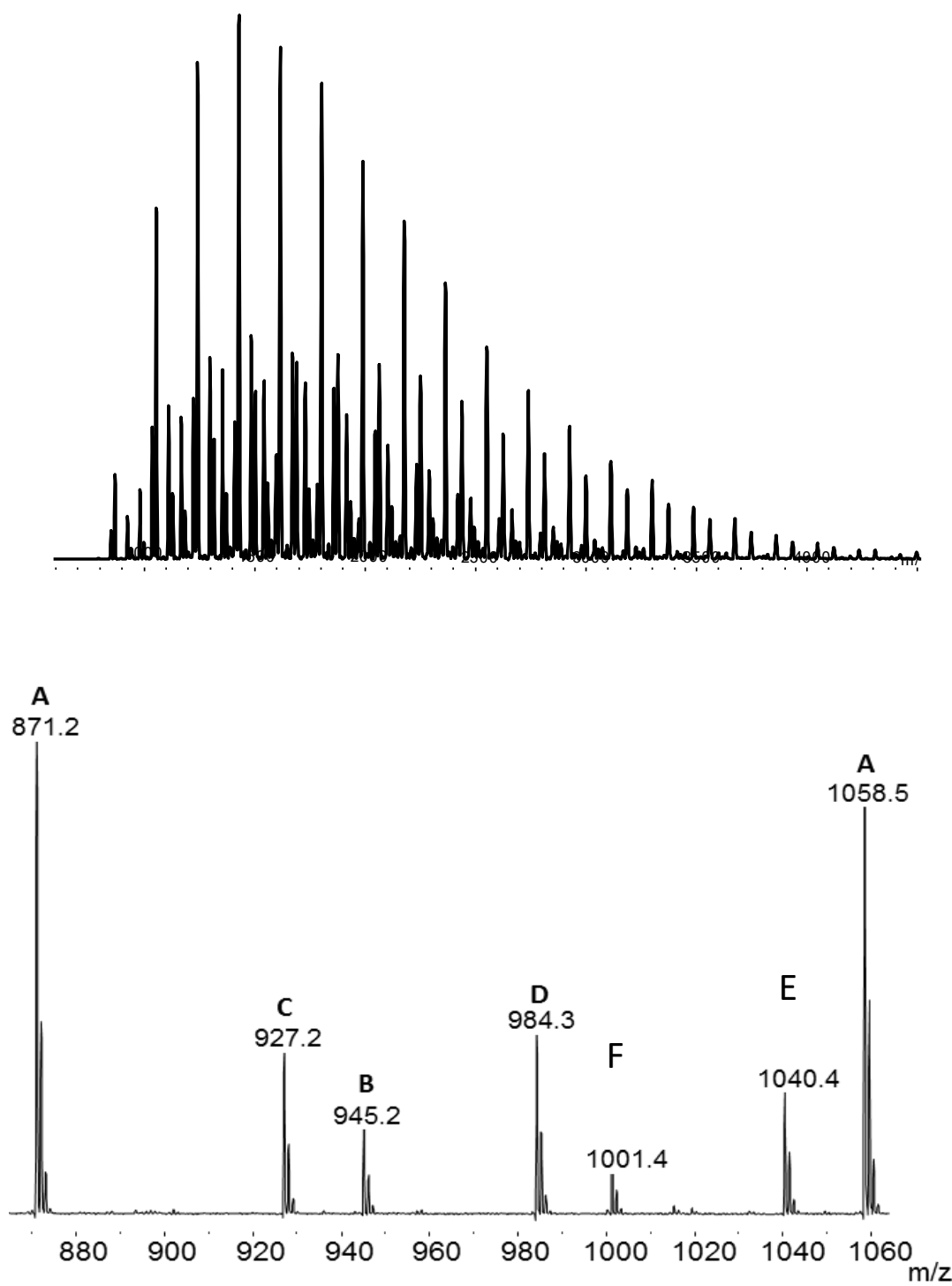

Figure S24: MALDI-TOF spectra (top: linear mode, bottom: reflectron mode) of poly(glycidyl N-dimethylcarbamate) initiated with DMAP (5 mol\%) (Table 2, entry 13) 


(n)

\begin{tabular}{|c|c|c|c|c|c|c|c|c|}
\hline Run & $\begin{array}{l}\overline{M_{n}}(\text { theo.) } \\
\left(\mathrm{g} \cdot \mathrm{mol}^{-1}\right)\end{array}$ & $\overline{D P}_{\text {(theo.) }}$ & Distribution & $\begin{array}{c}\overline{M_{n} \text { MALDI- }} \\
\text { ToF }^{\text {b }} \\
\left(\mathrm{g} \cdot \mathrm{mol}^{-1}\right)\end{array}$ & $\begin{array}{c}\overline{D P}_{\text {MALDI- }} \\
\text { ToF }^{\text {b }}\end{array}$ & $\begin{array}{c}\overline{M_{n}} \mathrm{SEC}^{\mathrm{c}} \\
\left(\mathrm{g} \cdot \mathrm{mol}^{-1}\right)\end{array}$ & $\begin{array}{l}\overline{M_{w}} \mathrm{SEC}^{\mathrm{c}} \\
\left(\mathrm{g} \cdot \mathrm{mol}^{-1}\right)\end{array}$ & $\bigoplus_{\mathrm{SEC}}$ \\
\hline \multirow{5}{*}{13} & \multirow{5}{*}{3850} & \multirow{5}{*}{20} & A & 2240 & 12 & \multirow{5}{*}{1430} & \multirow{5}{*}{2170} & \multirow{5}{*}{1.5} \\
\hline & & & B & 2470 & 13 & & & \\
\hline & & & $\mathrm{C}$ & 2050 & 11 & & & \\
\hline & & & $\mathrm{D}$ & 1900 & 10 & & & \\
\hline & & & $\mathrm{E}$ & 2180 & 12 & & & \\
\hline
\end{tabular}

Figure S25: MALDI-TOF and SEC results of poly(glycidyl $N$-dimethylcarbamate) initiated with DMAP (5 mol\%) (Table 2, entry 13) 


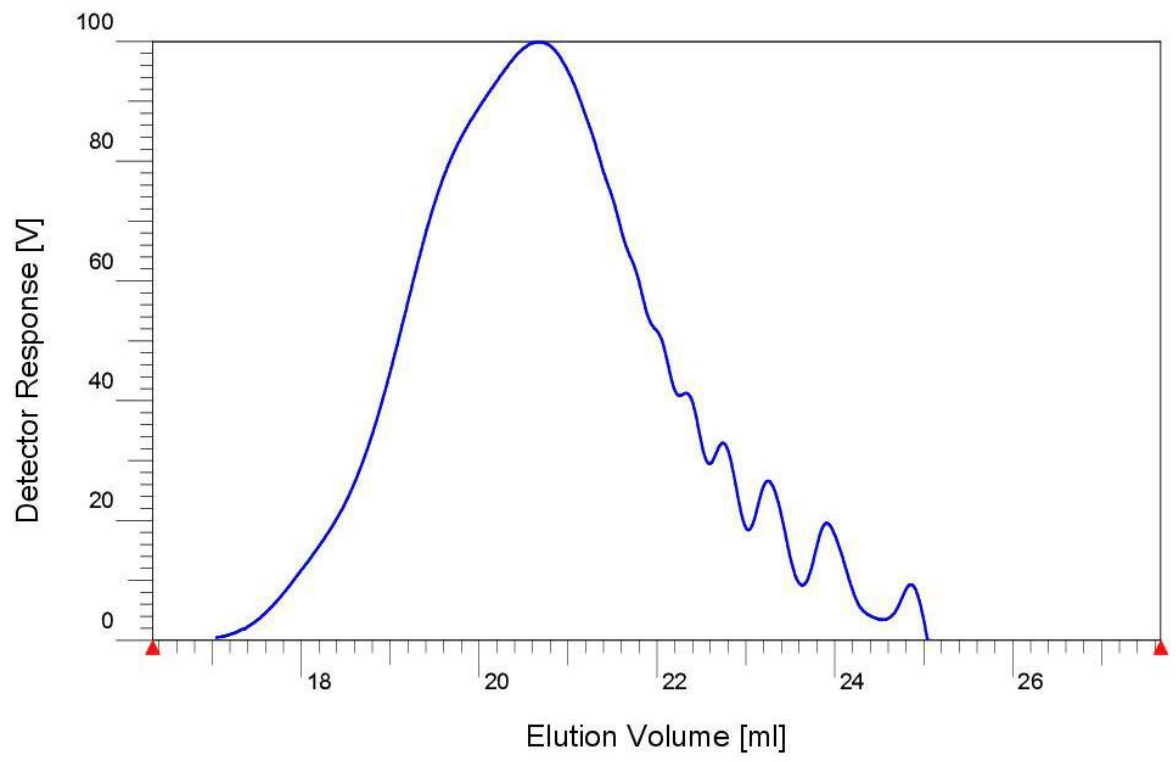

Figure S26: SEC trace of of poly(glycidyl $N$-dimethylcarbamate) initiated with DMAP (5 mol\%) (Table 2, entry 13) 


\section{SEC calibration}

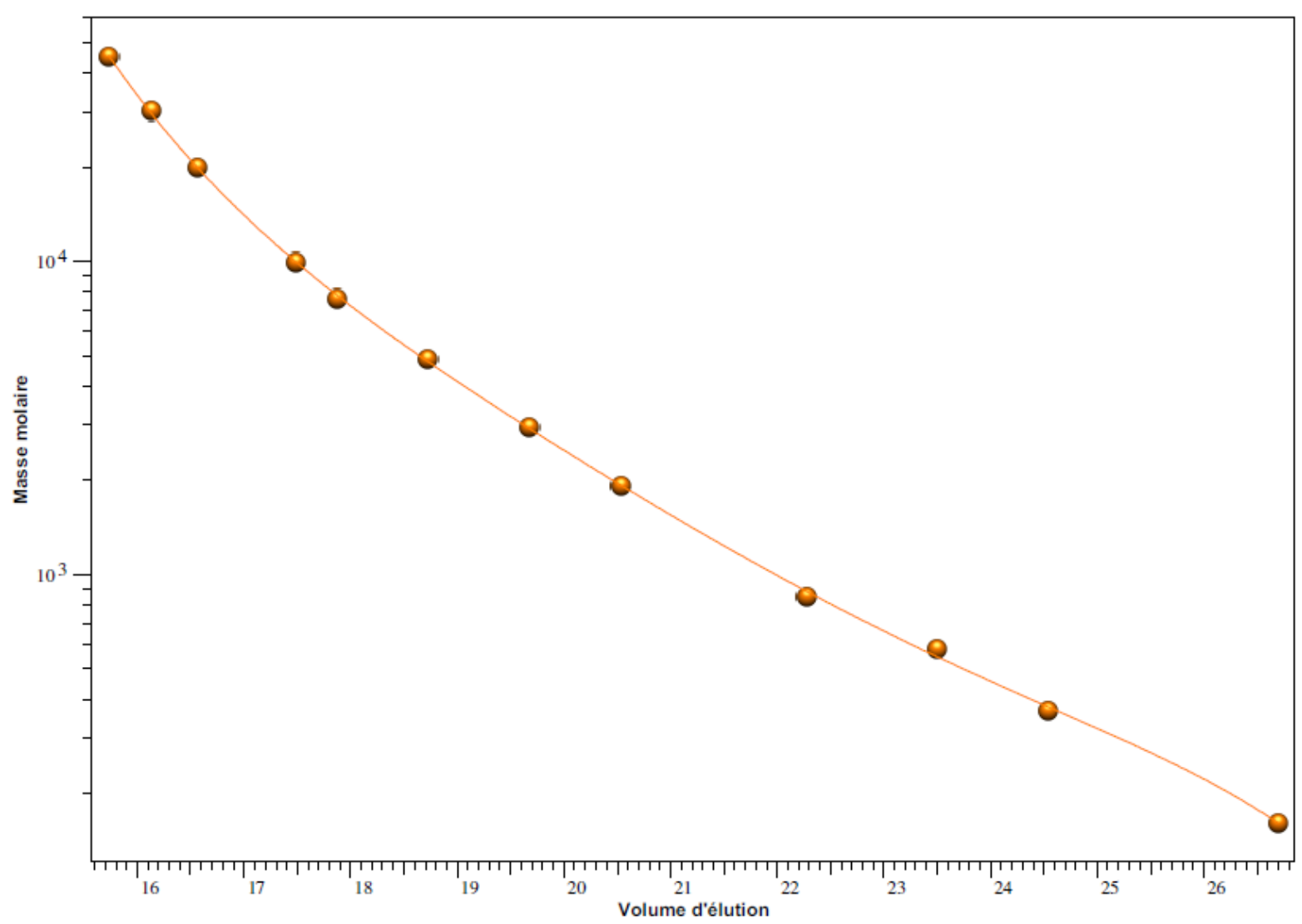

Figure S27 : SEC calibration curve.

\section{Conditions}

- Solvent : THF

- Temperature : $20^{\circ} \mathrm{C}$

- Concentration : 4.0 to $5.0 \mathrm{~g} / \mathrm{L}$

- Volume injection : $100 \mu \mathrm{L}$

- Colums : pre-column GPC Agilent $3 \mu \mathrm{m}$, ID 7,5 mm x $50 \mathrm{~mm}, 2$ column Mixed-E Agilent $3 \mu \mathrm{m}$, ID 7,5 mm x $300 \mathrm{~mm}, 1$ column 100A Agilent $3 \mu \mathrm{m}$, ID 7,5 mm x $300 \mathrm{~mm}$

- Rate : $1 \mathrm{~mL} / \mathrm{min}$

- Detection : RID

- Calibration : Polystyrene (fit polynomial 5) 
Poly(glycidyl carbamate) affinity with solvents

Example of calculation of HSP applied to poly(glycidyl $N, N$-dimethylcarbamate) using Hoftyzer and Van Krevelen method.

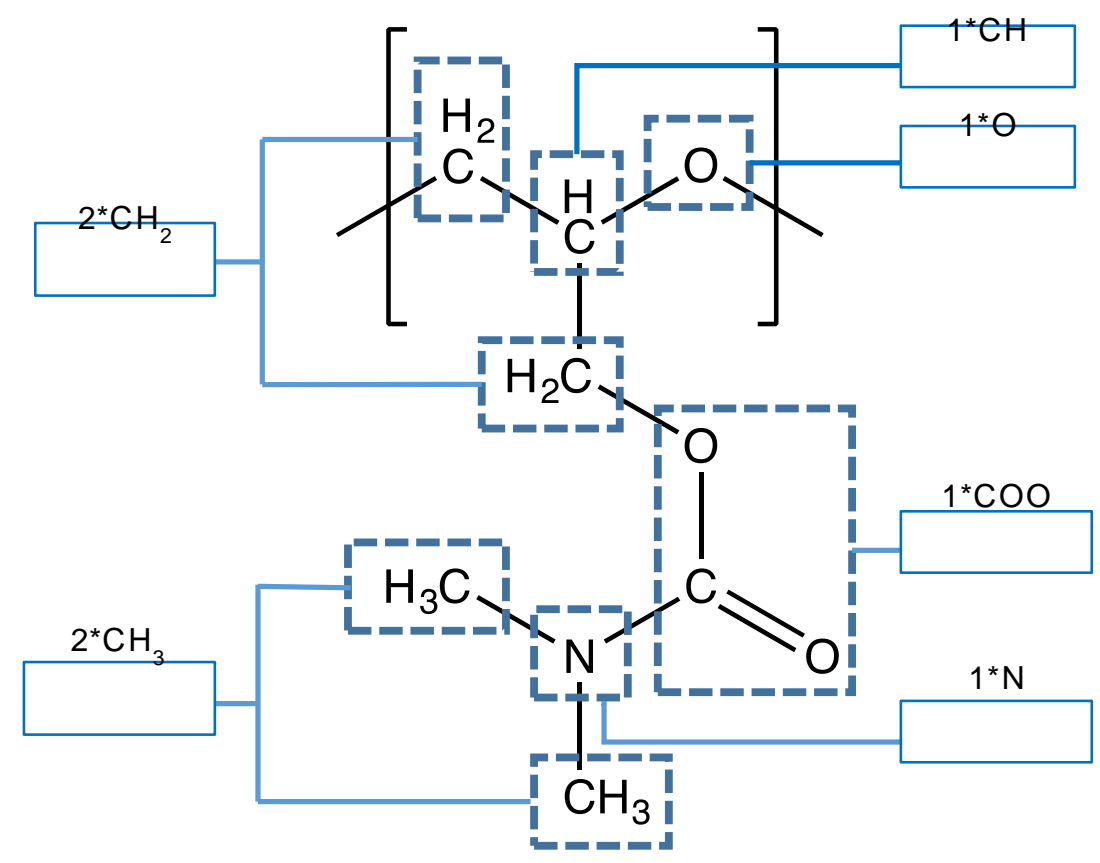

$$
\delta_{D}=\frac{\Sigma N_{i} F_{d i}}{V m} \quad \delta_{P}=\frac{\sqrt{\Sigma\left(N_{i} F_{p i}\right)^{2}}}{V m} \quad \delta_{H}=\sqrt{\frac{\Sigma N_{i} E_{h i}}{V m}}
$$

\begin{tabular}{cccccc}
\hline $\begin{array}{c}\text { Structural } \\
\text { groups }\end{array}$ & Count $\left(\mathrm{N}_{\mathrm{i}}\right)$ & $\begin{array}{c}\boldsymbol{N}_{\boldsymbol{i}} \times \boldsymbol{F}_{\boldsymbol{d i}} \\
\left(\mathrm{MJ} / \mathrm{m}^{3} \cdot \mathrm{mol}^{2}\right)^{1 / 2}\end{array}$ & $\begin{array}{c}\left(\boldsymbol{N}_{\boldsymbol{i}} \times \boldsymbol{F}_{\boldsymbol{~}}\right)^{2} \\
\left(\mathrm{MJ} / \mathrm{m}^{3} \cdot \mathrm{mol}^{2}\right)^{1 / 2}\end{array}$ & $\begin{array}{c}\boldsymbol{N}_{\boldsymbol{i}} \times \boldsymbol{E}_{\boldsymbol{h i}} \\
\mathrm{J} \cdot \mathrm{mol}^{-1}\end{array}$ & \\
\hline $\mathbf{C H}_{\mathbf{2}}$ & 2 & 540 & 0 & 0 & \\
$\mathbf{C H}$ & 1 & 80 & 0 & 0 & \\
$\mathbf{O}$ & 1 & 100 & 160000 & 3000 & \\
$\mathbf{C O O}$ & 1 & 390 & 240100 & 7000 & \\
$\mathbf{N}$ & 1 & 20 & 640000 & 5000 & \\
$\mathbf{C H}_{3}$ & 2 & 840 & 0 & 0 & $\boldsymbol{\delta}_{\boldsymbol{t}}$ \\
\hline & $\boldsymbol{V}_{\boldsymbol{m}}$ & $\boldsymbol{\delta}_{\boldsymbol{D}}$ & $\boldsymbol{\delta}_{\boldsymbol{p}}$ & $\boldsymbol{\delta}_{\boldsymbol{h}}$ & 21,5 \\
\hline
\end{tabular}




\begin{tabular}{|c|c|c|c|c|}
\cline { 2 - 5 } \multicolumn{1}{c|}{} & $\boldsymbol{\delta} \mathbf{D}$ & $\boldsymbol{\delta} \mathbf{P}$ & $\boldsymbol{\delta} \mathbf{H}$ & $\boldsymbol{\delta} \mathbf{t}$ \\
\hline Methanol & 15,1 & 12,3 & 22,3 & 29,6 \\
\hline Isthanol & 15,8 & 8,8 & 19,4 & 26,5 \\
\hline Ethyl acetate & 15,8 & 5,3 & 7,2 & 18,2 \\
\hline Tetrahydrofuran & 16,8 & 5,7 & 8 & 19,5 \\
\hline Acetone & 15,5 & 10,4 & 7 & 19,9 \\
\hline Dichloromethane & 18,2 & 6,3 & 6,1 & 20,2 \\
\hline Toluene & 18 & 1,4 & 2 & 18,2 \\
\hline Cyclohexane & 16,8 & 0 & 0,2 & 16,8 \\
\hline Diethyl ether & 14,5 & 2,9 & 5,1 & 15,6 \\
\hline p(GDMC) & 16,3 & 8,5 & 11,2 & 21,5 \\
\hline p(GMC) & 18,2 & 8,0 & 11,4 & 22,9 \\
\hline p(GPC) & 17,8 & 6,8 & 10,0 & 21,5 \\
\hline
\end{tabular}

Figure S28: HSP of various solvents and calculated form poly(glycidyl carbamates) 